\title{
O uso de frequencímetro para inferir indícios cognitivos em alunos adultos
}

Fabiana Santiago Sgobbi 1, Liane Margarida Rockenbach Tarouco 2, Fabrício Herpich 3

1Pós-Doutoranda em Informática na Educação - UFRGS - fabiana.sgobbi@ufrgs.br ${ }_{2}$ Professora titular da UFRGS, junto ao PPGIE - liane@penta.ufrgs.br

3 Doutorando em Informática na Educação - UFRGS - fabricio_herpich@hotmail.com

Resumo: Este artigo tem como objetivo investigar a existência de correlação entre a variabilidade da frequência cardíaca e o nível da atenção de estudantes durante a realização de tarefas que demandam grande processamento cognitivo, afim de constatar se existe causa e efeito das variáveis o suficiente para influenciar os indivíduos cognitivamente durante a execução das atividades. Os resultados indicaram aumento nas variações entre os três momentos dos testes, considerando análise dos dados coletados. Ocorreu aumento destes mesmos indicadores durante tarefas executivas, sugerindo aumento nos indícios cognitivos segundo a teoria de Thayer. Pode-se inferir que os usos de tais tecnologias são capazes de auxiliar a análise de indícios cognitivos reais em tempo de execução de uma determinada tarefa, visto que analisando os dados de amplas tecnologias tem-se o aumento esperado de atenção. Sugere-se relevante o desenvolvimento de estudos nesta área afim de criar uma ferramenta pratica e operacional capaz ajudar na escolha de um material didático mediante dados reais de cognição.

Palavras-chave: Cognição; Frequência Cardíaca; Atenção; Tecnologia.

\section{The use of Heart Rate Variability to infer cognitive cues in adult learners}

\begin{abstract}
This article aims to investigate the existence of a correlation between the heart rate variability and the level of attention of students during the tasks that demand great cognitive processing, in order to verify if there are cause and effect of the variables enough to influence individuals during the execution of activities. The results indicated an increase in the variations between the three moments of the tests, considering the analysis of the collected data. These same indicators increased during executive tasks, suggesting an increase in cognitive cues according to Thayer's theory. It can be inferred that the uses of such technologies are able to aid the analysis of real cognitive evidence at the time of execution of a given task, since analyzing the data of ample technologies, one has the expected increase of attention. It is suggested that the development of studies in this area be relevant in order to create a practical and operational tool capable of helping to choose a didactic material through real data of cognition.
\end{abstract}

Keywords: Cognition; Heart Rate; Attention; Technology.

\section{Introdução}

Existe demanda no setor educacional por tecnologias de informação e comunicação que auxiliem professores na tomada de decisão acerca da escolha de seus materiais educacionais que possam ensejar o ensino-aprendizagem de forma mais eficiente. Atualmente, tecnologias como sistemas tutores inteligentes, automação em salas de aulas, ambientes virtuais de aprendizado, ferramentas colaborativas, e Internet das Coisas vem 
apresentando um crescimento exponencial e assumindo um papel de destaque no âmbito educacional.

Neste contexto, outra tecnologia que têm se destacado no processo educacional consiste no uso de sensores, capazes de coletar dados em diferentes situações e que possibilitam inúmeras interpretações, inclusive para fins educacionais, tais como identificar os indícios cognitivos e nível de atenção do aluno. Dentre as possibilidades de identificação de indícios cognitivos, encontra-se a análise da Variabilidade de Frequência Cardíaca (VFC), que pode ser obtida com o uso de um frequencímetro (Thayer, 2007). Quintana et al. (2012) enfatiza que a VFC pode fornecer um novo marcador da capacidade de reconhecer as emoções nos seres humanos. Nesta perspectiva, Thayer et al. (2009) identificaram os dados que ligam o sistema nervoso central à VFC em humanos, o que possibilitou estabelecer que a VFC está intimamente relacionada com o cognitivo do ser humano.

Outros dispositivos sensores que podem contribuir para fins educacionais, consistem nas interfaces cérebro-computador, tais como as tecnologias de monitoramento eletroencefalográfico, as quais tem contribuído para identificar os níveis de atenção de estudantes, habilidade cognitiva que Ladewig (2000) destaca como sendo de grande importância, uma vez que a mesma "exerce uma função muito importante na capacidade de retenção de informações relevantes, pois é através dela, associada aos processos de controle, que guardamos informações na memória de longa duração" (Ladewig, 2000).

Esta pesquisa vem utilizar dispositivos de alta tecnologia. para identificar os aspectos indícios cognitivos do aluno, no intuito de auxiliar o professor, a adequar o conteúdo para um indivíduo ou para a sala como um todo. Assim, será possível monitorar a motivação e o engajamento dos alunos durante uma dada tarefa, permitindo que abordagens diferentes sejam elaboradas de acordo com as características destes estudantes para ensejar o aprendizado, abrindo uma nova possibilidade de planejamento e escolha de materiais didáticos. Nesta perspectiva, Schuh et al. (2016) afirmam que a neurociência tem apresentado pesquisas que envolvem o uso de interfaces cérebrocomputador (brain-computer interface - BCI), pois, além de apresentarem informações com altos níveis de acurácia, também são capazes de medir a atividade cerebral relacionada à intenção do usuário e traduzi-las em sinais de controle, que são detectados e decodificados por meio de aplicativos.

Com base nas contextualizações apresentadas, este artigo tem como objetivo verificar se dados da VFC e o nível de atenção aferidos concomitantemente podem inferir indícios cognitivo, com vistas a, em estudos futuros oferecer subsídios aos professores para adotar um material didático dependendo do envolvimento do aluno em determinado assunto. Para desempenhar esta pesquisa, foram utilizados dois testes (Efeito Stroop e Teste de Atenção Concentrada). Para monitorar a sequência de testes realizada, foram utilizados dois sensores: um frequencímetro capaz de oferecer dados sobre a Variabilidade de Frequência Cardíaca (Polar7); e um sensor de eletroencefalografia (EEG) que é capaz realizar a leitura das ondas cerebrais emitidas pelo córtex pré-frontal dorsolateral dos participantes no decorrer das atividades propostas pelos dois testes.

\subsection{A utilização da VFC como indícios cognitivos}

A variabilidade da frequência cardíaca é a medida do tempo entre dois batimentos cardíacos consecutivos, ou seja, é a mediada do tempo transcorrido entre dois intervalos $\mathrm{R}-\mathrm{R}$ consecutivos, medido através do eletrocardiograma (ECG). Este intervalo não é constante, ocorrendo variações no intervalo de tempo R-R (Thayer e Lane, 2000).

A frequência cardíaca é regulada instantaneamente por meio de mecanismos fisiológicos. Neste intervalo de tempo R-R, a frequência cardíaca é controlada pela 
atividade do sistema nervoso autônomo, através de seus ramos simpático e parassimpático. A atividade simpática reduz o intervalo R-R, enquanto a parassimpática o aumenta (Thayer et al., 2009). Na figura 1, tem-se o traçado eletrocardiográfico, evidenciando, qual espaço foi mensurado; trata-se do tempo entre os batimentos cardíacos.

Figura 1: Traçado eletrocardiográfico ( $\mathrm{VFC}=$ variabilidade de tempo entre $\mathrm{R}-\mathrm{R}$ )

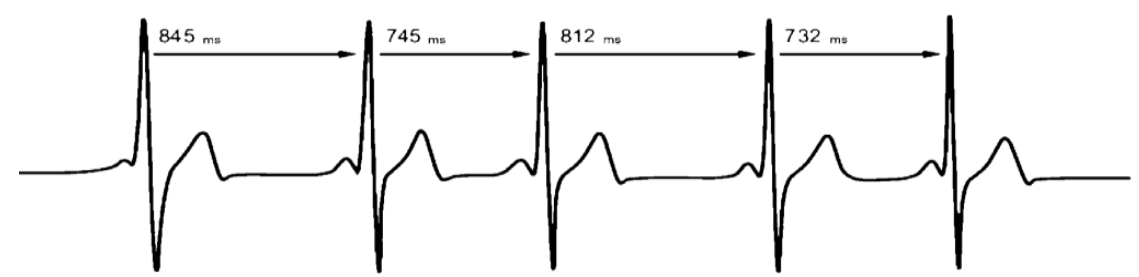

Fonte: Imagem do software Kubios que mede VFC

O cálculo da variabilidade da frequência cardíaca pode ser feito por dois métodos principais: o método estatístico dos intervalos R-R, chamado de domínio do tempo, e pela análise espectral de intervalos $\mathrm{R}-\mathrm{R}$, o espectro de densidade de potência. Esta variabilidade pode ser avaliada em curtos períodos (5 minutos ou menos), ou ainda durante 24 horas, usando o ECG (eletrocardiograma). Nesta pesquisa será utilizado o "delta" da frequência cardíaca, de cada segundo, dado por 60 dividido por Frequência Cardíaca, foi escolhido este método, por apresentar uma visão simplificada dos dados coletados. Visto que este artigo, tem como público a área de informática na educação.

Indicadores como a Variabilidade da Frequência Cardíaca tornam-se pertinentes, considerando como um método não invasivo e com acesso a fenômenos autonômicos por meio da interação entre a atividade simpática e parassimpática, causando modificações nos intervalos dos batimentos e nos componentes da frequência do coração. Alguns autores sugerem que baixa VFC relaciona-se com incidência de doença coronária, doenças vasculares, risco de declínio cognitivo e demência, alto nível de colesterol, alteração em pressão sanguínea e no cortisol; enquanto que elevada VFC está associada à saúde, flexibilidade cognitiva e desempenho favorável em funções executivas (Duschek et al., 2009; Britton et al., 2008).

Em contribuição, a teoria de Thayer (2009) (conhecido como modelo neurovisceral), propõe que todos estes processos de regulação: cognitivo, emocional e fisiológico são gerenciados pelo Sistema Nervoso Autônomo (SNA) e Sistema Nervoso Central (SNC) e correspondem à adaptação do organismo ao ambiente. A principal estrutura do córtex pré-frontal que inter-relaciona estes sistemas é o córtex cingulado anterior dorsal (cognitivo) e ventral (emocional). Como exemplo, durante o teste de Stroop o córtex cingulado anterior é ativado para a realização de tarefas que envolvem controle inibitório, do funcionamento executivo, enquanto que a regulação autonômica auxilia na adaptação do organismo ao ambiente na execução de atividades relacionadas a flexibilidade cognitiva (Matthews et al., 2004; Thayer et al., 2009). Além disso, existem conexões diretas e indiretas no coração ao Sistema Nervoso Autônomo e áreas do córtex, que formam uma importante relação entre a regulação dos ramos simpático e parassimpático, com respostas cardiovasculares e cognitivas (Thayer e Lane, 2009; Luft, Takase e Darby, 2009).

A inibição do sistema nervoso simpático e a ativação do sistema nervoso parassimpático estão associados com o aumento da VFC durante tarefas cognitivas executivas, e a uma das principais funções do córtex pré-frontal: a inibição da hiperativação do sistema nervoso simpático. A relação entre o bom desempenho em 
tarefas executivas e o aumento da VFC durante a realização dessas tarefas é discutida por pesquisas como de Luft, Takase, Darby (2009), Thayer e Lane (2009), Britton et al. (2008) e Hansen, Johnsen e Thayer (2003), havendo um consenso ao referir a que a ação do nervo vagal se relaciona com córtex pré-frontal (Luft, Takase e Darby, 2009).

Esta pesquisa vem corroborar com Luft, Takase, Darby (2009); Thayer e Lane (2009); Britton et al. (2008); Hansen, Johnsen, Thayer (2003) e outros no sentido de que quanto maior o VFC maior será a cognição e atenção (dado aferido no BCI).

\section{Metodologia}

Esta pesquisa de cunho qualitativo utilizou como estratégia o estudo de caso com propósito exploratório (Azevedo et al., 2009). Os participantes da pesquisa foram selecionados de forma probabilística e por acessibilidade ou conveniência, escolhidos intencionalmente, adultos de 21 a 26 anos de idade, com grau de instrução semelhante (todos cursando ensino médio tecnológico). Todos os indivíduos se autodeclararam saudáveis e ausentes de doenças neurológicas e psiquiátricas diagnosticadas até $o$ momento da coleta e sem uso contínuo de medicamentos psiquiátricos (como para depressão e ansiedade), Pesquisas de Kennedy e Raz (2009) e Tonkin (2009) afirmam que o uso do anti-hipertensivos e drogas cardiovasculares, podem modificar a VFC e estados cognitivos.

A participação foi inteiramente voluntária, com concordância registrada em Termo de Consentimento Livre e Esclarecido, conforme a resolução 196/96 do Conselho Nacional de Saúde. Foram selecionados 15 participantes, todavia devido à demora na aplicabilidade do teste, pelo número limitado de equipamentos, apenas 10 tiverem tempo hábil para realizarem o teste. Esta coleta de dados se deu na biblioteca de uma instituição de ensino, de forma individual.

Foram escolhidos dois testes, o "Efeito Stroop" e "Teste de Atenção Concentrada", esta escolha derivou da necessidade de trabalharmos indícios cognitivos. Segundo Coutinho et al. (2006) a atenção é um fenômeno complexo e multifacetado, cujos limites se interseccionam com a percepção, a memória, a motivação, cognição e o nível de consciência, dentre outros. O processo de aprendizagem exige um certo nível de ativação e atenção, de vigilância e seleção das informações. A ativação, por meio da vigilância, conecta-se com a atenção, no sentido da capacidade de focalização da atividade. Esses são elementos fundamentais de toda atividade neuropsicológica, essenciais para manterem as atividades cognitivas.

\subsection{As tecnologias}

\section{O monitor cardíaco ou frequencímetro}

O monitor cardíaco é um instrumento sensível à atividade elétrica gerada pela contração do miocárdio, captando e transmitindo sinais para um dispositivo visualizador da atividade cardíaca. O modelo A300 da Polar é composto de 2 unidades: uma unidade de visualização e armazenamento de dados (em formato de relógio) e uma unidade de aquisição e transmissão de dados (sensor H7) que fica plugado em uma cinta, que possui dois eletrodos e é a parte que fica em contato direto com o sujeito.

O sensor capta a contração do músculo cardíaco e enviam para o componente transmissor. Este componente envia então os tempos entre cada contração muscular (intervalo R-R) para a unidade de armazenamento e visualização (no formato de um relógio). Esta unidade irá armazenar os dados de toda a coleta para posteriormente enviar as informações através de bluetooth para um computador. 


\section{Brain-Computer Interface (BCI)}

Para capturar o nível de atenção dos participantes foi utilizado o sensor MindWave Mobile 2 da fabricante NeuroSky (2019), que consiste em um headset que registra dados eletroencefalográficos brutos através de um único sensor usado em contato com a região do córtex pré-frontal dorsolateral do participante. Por meio do registro dessas informações, o sensor é capaz de fornecer duas medidas personalizadas sobre o participante que está equipado com o sensor, que correspondem a atenção e meditação, variando de 0 a 100, denotando o nível de foco do aluno (Herpich et al., 2018).

Em relação a captura os dados emitidos pelo sensor MindWave 2, foi utilizado o aplicativo Mindful Metrics (2019), que além de realizar a conexão com o sensor (via bluetooth), também possibilita a geração e visualização de gráficos, o compartilhamento dos resultados e a exportação dos dados em formato compatível para posterior análise estatística, facilitando o acesso aos dados coletados.

\section{2 - Procedimento}

Logo após a pesquisadora explicar os objetivos da pesquisa, os participantes foram equipados com os dois sensores simultaneamente (frequencímetro e o brain-computer interface), A pesquisa foi realizada individualmente, em uma sala da biblioteca da instituição, local onde na ocasião da coleta de dados, se encontrava vazio (sem a presença de alunos).

A execução das tarefas se deu de forma sequencial primeiro o participante fez o Teste Stroop e depois o Teste de Atenção Concentrada. A coleta de dados se deu de forma contínua (por teste), todavia foi anotado pela pesquisadora (três momentos): segundos antes de iniciar o teste (momento1), o início do teste propriamente dito (momento2) e segundos após o término do teste (momento3). Esta divisão se deu para que houvesse parâmetros de dados em 3 estágios, o mesmo procedimento foi tomado para coleta de dados na execução dos dois testes.

Cada teste demorou em torno de 2 a 3 minutos, todavia houve uma grande demora, por parte dos participantes em colocar os sensores, as meninas iam até o banheiro para colocar a cinta com o sensor A7, na região do tórax, e ambos os sexos demoravam para se adaptarem ao BCI. Um fator que deve ser evidenciado foi a grande empolgação e curiosidade com o uso dos sensores.

O Teste Stroop é baseado na observação de que os indivíduos podem ler palavras muito mais rápido do que podem identificar e nomear as cores. Segundo Golden (1978) a dimensão cognitiva aproveitada pelo Stroop está associada à flexibilidade cognitiva, resistência à interferência de estímulos externos, criatividade e psicopatologia - todos os quais influenciam a capacidade do indivíduo de lidar com o estresse cognitivo e processar informações complexas. O teste foi usado como por sua administração rápida e fácil e ser validado por diversas pesquisas (Thayer et al., 2009; Williams et al., 1996).

Figura 2: Tela inicial do Teste Stroop

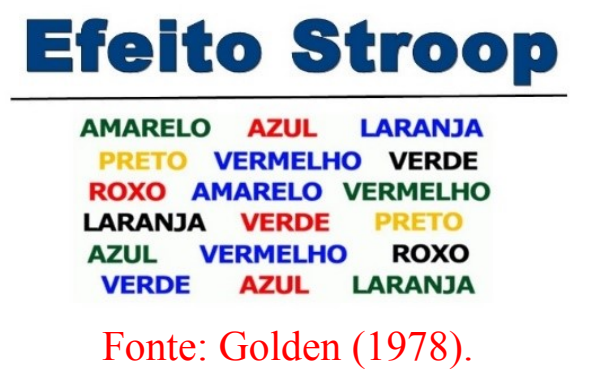


A respeito do segundo teste, Cecilio-Fernandes (2007) explica que o Teste de Atenção Concentrado (TEACO-FF) fornece uma medida da atenção concentrada da pessoa, que pode ser obtida pelo resultado dos estímulos que a pessoa deveria marcar e marcou, subtraído dos erros (estímulos que não deveriam ser marcados e foram) e das omissões (estímulos-alvo que não foram marcados pela pessoa). $\mathrm{Na}$ sua totalidade o instrumento possui 441 estímulos (conforme visto na figura 3 ) distribuídos em 21 colunas com 21 estímulos cada. Do total, 140 são estímulos-alvo, e cada coluna contém nove alvos e 14 estímulos distratores. $O$ teste de atenção concentrada informa sobre a capacidade de foco em uma determinada tarefa em detrimento de outras, levando em conta o tempo de duração. Na figura 3 tem-se a tela do teste de atenção concentrada.

Figura 3: Tela do teste de atenção concentrada

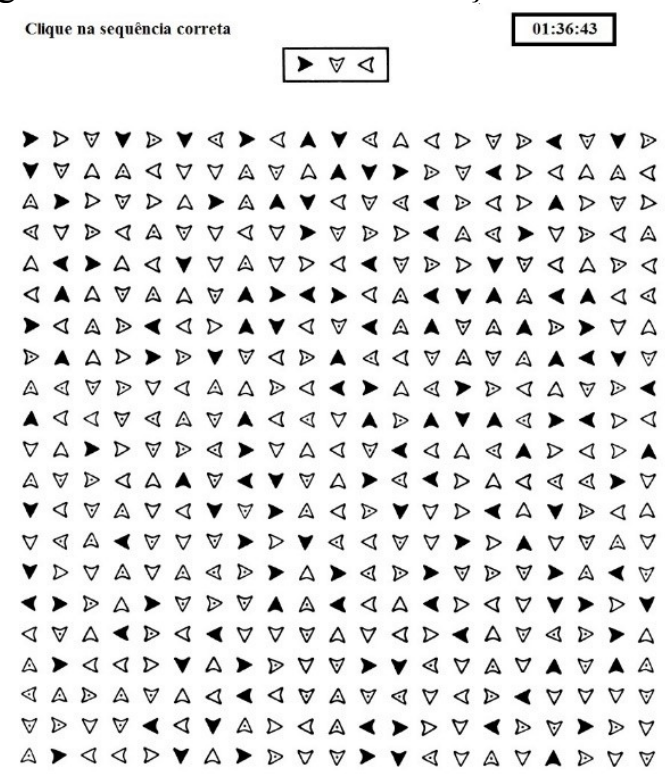

Fonte: Cecilio-Fernandes (2007).

\section{Resultado}

Durante a etapa de análise de dados foram utilizados diversos softwares. A descrição e a finalidade de cada um no escopo da pesquisa estão explicitadas a seguir:

O software Polar Flow acompanha o monitor cardíaco Polar A 300 e tem por finalidade receber, via bluetooth, os dados da unidade de armazenamento e exportar os intervalos de tempo entre cada batimento cardíaco (FC) para análise. Ele gera os dados brutos do tempo de cada intervalo FC no formato de uma coluna com $\mathrm{n}$ linhas, sendo $\mathrm{n} o$ número de intervalos entre batimentos durante a coleta.

A primeira fase do procedimento de análise de dados consistiu na elaboração de planilha geral da pesquisa no software MS Excel, tal planilha contém a Frequência Cardíaca e os dados do BCI, dados coletados durantes os dois testes.

$\mathrm{O}$ processamento dos dados do delta da FC aconteceu em duas fases. A primeira através do software Polar Flow que recebeu os dados gerados pelo monitor cardíaco e exportou um documento contendo a sequência de intervalos FC de cada sujeito, calculado do Delta da FC e suas respectivas médias de cada um dos três momentos: antes do teste (momento1), durante o teste (momento2) e após o teste (momento3) de cada participante, em cada teste. 
Os dados do BCI, foram coletados pelo aplicativo Mindful Metrics através do sensor MindWave 2 e exportado para uma planilha do MS Excel, processados para que as análises estatísticas fossem feitas, dados que serão apresentados nas próximas seções.

\subsection{Análise do delta FC durante a execução de cada teste.}

$\mathrm{Na}$ tabela abaixo temos os dados, coletados durante os três momentos, nos dois testes.

Tabela 1: Dados obtidos através do frequencímetro (nos testes)

\begin{tabular}{|l|c|c|c|c|c|c|}
\hline & \multicolumn{3}{|c|}{ Teste de Stroop } & \multicolumn{2}{c|}{ Teste de Atenção Concentrada } \\
\hline $\mathrm{N}^{\mathbf{o}}$ & MDdF1 & MDdF2 & MDdF3 & MDdF1 & MDdF2 & MDdF3 \\
\hline 1 & 0,6575 & 0,7090 & 0,6772 & 0,7249 & 0,8550 & 0,6828 \\
\hline 2 & 0,6948 & 0,7070 & 0,6980 & 0,7142 & 0,7693 & 0,7321 \\
\hline 3 & 0,6815 & 0,6861 & 0,6883 & 0,6914 & 0,7631 & 0,7160 \\
\hline 4 & 0,6916 & 0,7272 & 0,6762 & 0,6861 & 0,7878 & 0,6667 \\
\hline 5 & 0,6672 & 0,7474 & 0,6624 & 0,6768 & 0,7553 & 0,7338 \\
\hline 6 & 0,6570 & 0,7820 & 0,6776 & 0,6701 & 0,7906 & 0,6940 \\
\hline 7 & 0,6399 & 0,7972 & 0,6812 & 0,6933 & 0,8317 & 0,7121 \\
\hline 8 & 0,6588 & 0,8012 & 0,6774 & 0,6589 & 0,7230 & 0,6898 \\
\hline 9 & 0,6473 & 0,7614 & 0,6881 & 0,6453 & 0,7448 & 0,6819 \\
\hline 10 & 0,6532 & 0,7632 & 0,6634 & 0,6667 & 0,7318 & 0,6933 \\
\hline
\end{tabular}

Legenda

$\mathrm{N}^{\mathrm{o}}$ - número do participante

MDdF1 - Média Delta FC momento 1

MDdF2 - Média Delta FC momento 2

MDdF3 - Média Delta FC momento 3

Ao analisarmos o Teste Stroop, verificamos uma significativa variação, entre as médias do Delta da FC, em relação a MDdF1 e MDdF2, o mesmo acontece de MDdF2 em relação a MDdF3. Calculando estas variações, entre a primeiro momento e a segundo momento, fica evidenciado um aumento médio de $12 \%$, no total dos participantes. Sendo que apenas $20 \%$ ficaram com um aumento abaixo de $9 \%$. O mesmo ocorreu, em relação às variações, do momento 2 para o momento 3 houve uma queda do delta $\mathrm{FC}$ em média de $8 \%$, sendo $90 \%$ por participantes tiveram dados menos que $5 \%$ de variação.

Os testes de atenção concentrada, vem corroborar com os dados do Teste Stroop, onde do momento 1, para momento2, ocorrei um aumento médio (entre todos os participantes) de $11 \%$, variando de $6 \%$ a $17 \%$. No momento 2 , para momento 3 , os dados elucidam uma variação média menor, o que se justifica por se tratar no momento final do teste, tivemos uma variação média de $7 \%$. Os dados permitiram primeiramente indicar que os diferentes momentos produziram respostas diferentes estatisticamente. Segundo Thayer (2007) a VFC efetivamente varia em função das diferentes tarefas, onde quanto maior a VFC maior o nível de cognição.

\subsection{Análise dos dados do BCI.}

A análise dos dados do BCI, não será fracionada em momentos, isso porque o software do fabricante Nerousky, não permite que façamos delimitações de tempo (minuto/ segundo), são fornecidos apenas dados de atenção e meditação sem a correlação com o tempo ocorrido. Nesta análise utilizaremos apenas os dados de atenção. Na tabela abaixo temos os dados dos 10 participantes, a porcentagem de tempo do total gasto no teste, que o participante atingiu a determinado número de pontos de atenção (Tabela 2). 
Tabela 2: Dados do BCI

\begin{tabular}{|c|c|c|c|c|c|c|c|c|}
\hline & \multicolumn{4}{|c|}{ Teste Stroop } & \multicolumn{3}{c|}{ Teste de Atenção Concentrada } \\
\hline $\mathrm{N}^{\mathrm{o}}$ & 76 a 100 & 51 a 75 & 26 a 50 & 1 a 25 & 76 a 100 & 51 a 75 & 26 a 50 & 1 a 25 \\
\hline 1 & $4,71 \%$ & $28,25 \%$ & $51,76 \%$ & $15,28 \%$ & $5,97 \%$ & $52,56 \%$ & $32,86 \%$ & $8,61 \%$ \\
\hline 2 & $8,10 \%$ & $44,44 \%$ & $38,82 \%$ & $8,64 \%$ & $7,98 \%$ & $53,89 \%$ & $29,98 \%$ & $8,15 \%$ \\
\hline 3 & $7,31 \%$ & $50,89 \%$ & $33,78 \%$ & $8,02 \%$ & $6,78 \%$ & $29,87 \%$ & $49,98 \%$ & $13,37 \%$ \\
\hline 4 & $3,01 \%$ & $26,67 \%$ & $54,22 \%$ & $16,10 \%$ & $6,10 \%$ & $56,23 \%$ & $32,89 \%$ & $4,78 \%$ \\
\hline 5 & $5,12 \%$ & $46,41 \%$ & $38,62 \%$ & $9,85 \%$ & $3,78 \%$ & $54,12 \%$ & $35,98 \%$ & $6,12 \%$ \\
\hline 6 & $9,12 \%$ & $53,09 \%$ & $31,39 \%$ & $6,40 \%$ & $8,15 \%$ & $52,01 \%$ & $33,12 \%$ & $6,72 \%$ \\
\hline 7 & $8,22 \%$ & $57,36 \%$ & $27,50 \%$ & $6,92 \%$ & $4,01 \%$ & $49,92 \%$ & $35,87 \%$ & $10,20 \%$ \\
\hline 8 & $10,89 \%$ & $56,76 \%$ & $26,87 \%$ & $5,48 \%$ & $9,54 \%$ & $50,87 \%$ & $30,98 \%$ & $8,61 \%$ \\
\hline 9 & $9,02 \%$ & $53,14 \%$ & $31,65 \%$ & $6,19 \%$ & $6,42 \%$ & $51,87 \%$ & $35,89 \%$ & $5,82 \%$ \\
\hline 10 & $9,65 \%$ & $52,87 \%$ & $33,00 \%$ & $4,48 \%$ & $8,67 \%$ & $52,54 \%$ & $28,97 \%$ & $9,82 \%$ \\
\hline
\end{tabular}

Fonte: cálculos de percentagem de atenção do BCI

No Teste de Stroop, ao compararmos o nível de atenção dos participantes temos que $80 \%$ dos participantes tiveram aproximadamente $50 \%$ do tempo total do teste de 51 a 75 pontos de atenção e apenas 2 participantes tiveram mais de $10 \%$ do tempo com pouca atenção. Dados que corroboram com os dados do frequencímetro, embasados na teoria de Thayer(2009), apontando para um aumento de indícios cognitivos.

Ao analisarmos os dados referente ao teste Stroop, percebemos que um nível alto de atenção, na maior parte do tempo de execução do teste, houve um nível de atenção de 51 a 75 pontos, dados que corrobora com a teoria do Efeito Stroop, de acordo com Matthews, et al. (2004), durante o teste de Stroop o córtex cingulado anterior é ativado para a realização de tarefas que envolvem controle inibitório, do funcionamento executivo, enquanto que a regulação autonômica auxilia na adaptação do organismo ao ambiente na execução de atividades relacionadas a flexibilidade cognitiva (Thayer, et al 2009).

Quando voltamos o olhar aos dados do teste Atenção Concentrada, temos aproximadamente $90 \%$ dos participantes com nível alto de atenção, dados com contribuem para identificar indícios cognitivos neste período, visto que existem conexões diretas e indiretas no coração ao Sistema Nervoso Autônomo e áreas do córtex, que formam uma importante relação entre a regulação dos ramos simpático e parassimpático, com respostas cardiovasculares e cognitivas (Thayer e Lane, 2009; Luft, Takase e Darby, 2009).

\section{Conclusão e discussões}

Os resultados desta pesquisa sugerem que as variações dos dados vindo do frequencímetro, acompanharam as mudanças de estimo nos três momentos do teste. Segundo Thayer (2007) o aumento da VFC está associado ao aumento da atividade do sistema nervoso simpático e menor resposta do sistema nervoso parassimpático. Isso sugere que, o córtex cerebral participa ativamente da regulação da atividade autonômica durante a vigília. Esse fenômeno é evidente quando se vai do estado de repouso para uma demanda cognitiva, observacional, concentração e memória (Thayer, 2007). Pode-se inferir, as mudanças de atividades cognitivas influenciam diretamente a Variabilidade de Frequência Cardíaca, para corroborar com tais dados verificamos um aumento da atenção, durante a execução dos testes, aferido pelo BCI.

Segundo Brosschot, Verkuil e Thayer (2010) quando ocorre perseveração cognitiva sugere-se aumento de ativação simpática. De tal modo, a tomada de decisão 
pode ser regulada pela redução da ação do sistema nervoso simpático e aumento da atividade do nervo vago, desempenhando um papel na flexibilidade cognitiva e controle inibitório (Ohira et al.,2010).

Conclui-se que a variabilidade da frequência cardíaca são instrumentos valiosos capazes evidenciar indícios cognitivos durante a execução de tarefas. É fato que por se tratar de uma área pouco explorada e tão importante, se faz necessário mais pesquisas para consolidar e viabilizar esta hipótese.

O próximo passo da pesquisa é aumentar o número de participantes e observar com a execução de outras atividades, de forma a validar a teoria com um modelo de dados sólido o suficiente para embasar o desenvolvimento de uma ferramenta que possa futuramente validar e utilizar técnicas computacionais para a recomendação de materiais didáticos com base em dados cientificamente comprovados.

\section{Referências}

AZEVEDO, B. F. T.; REATEGUI, E. BERNI; BEHAR, PATRICIA ALEJANDRA. Estudo de análise qualitativa em fórum de discussão. RENOTE: revista novas tecnologias na educação [recurso eletrônico]. Porto Alegre, RS, 2009.

BRITTON, A, SINGH-MANOUX, A., HNATKOVA, K., MALIK, M., MARMOT, M. G., \& SHIPLEY, M.. The Association between Heart Rate Variability and Cognitive Impairment in Middle-Aged Men and Women. The Whitehall II Cohort Study. Neuroepidemiology, 31(2), 115-121, 2008.

BROSSCHOT, J.F., VERKUIL, B., \& THAYER, J.F. Conscious and unconscious perseverative cognition: Is a large part of prolonged physiological activity due to unconscious stress? Journal of Psychosomatic Research. ARTICLE IN PRESS, accepted 2 February 2010.

CECILIO-FERNANDES, DARIO; MARÍN RUEDA, FABIÁN JAVIER. Evidência de validade concorrente para o Teste de Atenção Concentrada (TEACO-FF). Psic: revista da Vetor Editora, v. 8, n. 2, p. 167-174, 2007.

COUTINHO G, ARAÚJO C. Subtipos clínicos de TDAH e seus achados neurobiológicos. In: Valle LELR, ed. Neuropsiquiatria: infância e adolescência. ABENEPI. Rio de Janeiro: Wak; 2006.

DUSCHEK, S., MUCKENTHALER, M., WERNER, N., \& PASO, G. A. R. . Relationships between features of autonomic cardiovascular control and cognitive performance. Biological Psychology 81, 110-117, 2009.

Golden, Charles J.; Freshwater, Shawna M. Stroop color and word test. 1978.

HANSEN, A. L., JOHNSEN, B. H., THAYER, J. F.. Vagal influence on working memory and attention. International Journal of Psychophysiology, 48, 263-274, 2003.

HERPICH, F.; GUARESE, R. L. M.; CASSOLA, A. T.; AND TAROUCO, L. M. R.. "Mobile Augmented Reality impact in Student Engagement: an Analysis of the Focused Attention dimension". International Conference on Computational Science and Computational Intelligence (CSCI), 2018.

KENNEDY, K. M., RAZ, N. . Aging white matter and cognition: Differential effects of regional variations in diffusion properties on memory, executive functions, and speed. Neuropsychologia, 47, 916-927, 2009.

LADEWIG, I. A importância da atenção na aprendizagem de habilidades motoras. Revista paulista de Educação Física, 20 (3), p. 62-71, 2000. 
LONG X, FONSECA P., FOUSSIER J, HAAKMA R, AARTS R., Sleep,Wake classification with actigraphy and respiratory effort using dynamic warping, IEEE J.Biomed.Health Inform.18,1272-1284, 2014.

LUFT, C. D. B, TAKASE, E., DARBY, D. . Heart rate variability and cognitive function: Effects of physical effort. Biological Psychology, 82, 196-201, 2009.

QUINTANA, D. S., GUASTELLA, A. J., OUTHRED, T., HICKIE, I. B., KEMP, A. H. Heart rate variability is associated with emotion recognition: Direct evidence for a relationship between the autonomic nervous system and social cognition. International Journal of Psychophysiology, 86, 168-172, 2012.

MATTHEWS, S. C., PAUluS, M. P., SimMOnS, A N., NElESEN, R. A., \& DIMSDALE, J. E. . Functional subdivisions within anterior cingulate cortex and their relationship to autonomic nervous system function NeuroImage, 22, 11511156, 2004.

Mindful Metrics. (2019). Página Oficial da Ferramenta. Disponível em: < http://www.ufrgs.br/avatar >. Acesso em: Abril de 2019.

Página Oficial da Ferramenta. Disponível em: <http://store.neurosky.com/ products/mindwave-mobile>. Acesso: Abril de 2019.

OHIRA, H., ICHIKAWA, N., NOMURA, M., ISOWA, T., KIMURA, K., KANAYAMA, N., FUKUYAMA, S., SHINODA, J., YAMADA, J. Brain and autonomic association accompanying stochastic decision-making, Neurolmage, 49(1), p. 1024-37, 2009.

SCHUH, A, CAMPOS, M. D. B., BEZ, M. MOSSMANN, J. B. . "Usability Evaluation of a Wheelchair Virtual Simulator Controlled by a Brain-Computer Interface: Lessons Learned to the Design Process". Universal Access in Human-Computer Interaction. Interaction Techniques and Environments, 9738. Antona M. \& Stephanidis, C. (eds). Cham - Switzerland: Springer International Publishing, p. 92- 101, 2016.

THAYER, J. F. . What the heart says to the brain (and vice versa) and why we should listen.Psychological Topics, 16(2), 241-250, 2007.

THAYER, J. F. Heart rate variability: a neurovisceral integration model. 2009.

THAYER, J. F., HANSEN, A. L., SAUS-ROSE, E., \& JOHNSEN, B. H . Heart rate variability, prefrontal neural function, and cognitive performance: the neurovisceral integration perspective on self-regulation, adaptation, and health. Annals of Behavioral Medicine, v. 37, n. 2, p. 141-153, 2009.

THAYER, JULIAN F.; LANE, RICHARD D. A model of neurovisceral integration in emotion regulation and dysregulation. Journal of affective disorders, 61 (3), p. 201216, 2000.

THAYER, JULIAN F.; LANE, RICHARD D. Claude Bernard and the heart-brain connection: Further elaboration of a model of neurovisceral integration. Neuroscience \& Biobehavioral Reviews, v. 33, n. 2, p. 81-88, 2009.

TONKIN, A.L. Autonomic Dysfunction: Drug Induced. Encyclopedia of Neuroscience, $809815,2009$.

WILLIAMS, J. MARK G.; MATHEWS, ANDREW; MACLEOD, COLIN. The emotional Stroop task and psychopathology. Psychological bulletin, 120 (1), p. 3, 1996. 